\title{
Evaluation of Salivary Levels of Proinflammatory Cytokines (IL- 1 $\alpha$, IL-8 and GM-CSF) in Adult Orthodontic Patients
}

\author{
Batool H. Al-Ghurabi ${ }^{1}$, Harraa S. Mohammed-Salih ${ }^{2}$, Ata'a Ghazi ${ }^{3}$, \\ Hayder Fadhil Saloom ${ }^{4}$ \\ 1Assist. Prof. of Microbiology-Immunology/B.Sc.,M.Sc., PhD. (Basic Science Department, College of Dentistry/ \\ Baghdad University, Baghdad, Iraq). \\ 2Assistant lecturer of Orthodontics/ B.D.S., M.Sc. (Orthodontic Department, College of Dentistry/ Baghdad \\ University, Baghdad, Iraq). \\ 3Assistant lecturer of Orthodontics/ B.D.S., M.Sc. (Orthodontic Department, College of Dentistry/ Al- \\ Mustansiriya University, Baghdad, Iraq). \\ 4Prof. of Orthodontics/ B.D.S., M.Sc. (Orthodontic Department, College of Dentistry/ Baghdad University, \\ Baghdad, Iraq).
}

\begin{abstract}
Background: Orthodontic tooth movement is a type of tissue injury leading to an inflammatory response. Cytokines are involved in initiating, amplifying, perpetuating, and resolving inflammatory responses. The aim of the present study was to investigate the effects of orthodontic tooth movement on the periodontium by analyzing proinflammatory cytokines (IL-1 $\alpha, I L-8$ and GM-CSF) levels within saliva.

Subjects and Methods: Saliva samples were collected from 18 subjects (12 females and 6males) at baseline time (BT), after 2 weeks (T2) and after 4weeks (T4) from placement of orthodontic appliance. Salivary IL-1 $\alpha$, IL8 and GM-CSF were measured by means of enzyme-linked immune-sorbent assay.

Results: The current results revealed no statistically significant differences in the levels of IL-1 $\alpha$ at any times, $P>0.05$. On the other hand, significant elevation in median salivary levels of IL-8 and GM-CSF was found after 2 weeks and after 4 weeks from placement of orthodontic appliance compared to their levels at baseline time, $P<0.01$. In addition the levels of these cytokines were significantly decrease at 4 weeks when compared to their levels at 2 weeks, $P<0.01$.

Conclusion: These findings suggest that proinflammatory cytokines play a crucial role in bone resorption after the application of orthodontic force.
\end{abstract}

Key words: Orthodontic tooth movement, Cytokines.

\section{Introduction}

Orthodontic tooth movement is based on force induced periodontal ligament and alveolar bone remodeling. Mechanical stimuli exerted on a tooth cause an inflammatory response in the periodontal tissue. Inflammatory mediators trigger the biological processes associated with alveolar bone resorption and apposition. An important breakthrough in bone biology was the identification of the role of cytokines in bone remodeling ${ }^{(1,}$ ${ }^{2}$. Cytokines are involved in initiating, amplifying, perpetuating, and resolving inflammatory responses. They are key mediators for tissue damage and play an important role in tooth movement ${ }^{(3)}$. The released cytokines evoke cellular responses in the various cell types in and around teeth, providing a favorable microenvironment for tissue deposition or resorption. Various cell-signaling pathways are activated, which ultimately stimulate periodontal ligament turnover, as well as localized bone resorption and bone deposition ${ }^{(4,5)}$.

Interleukin-1 alpha (IL-1 $\alpha$ ) is a polypeptide cytokines produced primarily by cells of the mononuclear phagocytic lineage. Target cell type includes osteoblasts ${ }^{(6)}$. In general, IL-1 is responsible for the production of inflammation, as well as plays one of the central roles in the regulation of the immune responses ${ }^{(7)}$. IL- 8 is a potent proinflammatory cytokine that has a key role in the recruitment and activation of neutrophils during inflammation. It is secreted mainly by monocytes and is important in regulating alveolar bone resorption during tooth movement by acting early in the inflammatory response ${ }^{(8)}$. Granulocyte-macrophage colony-stimulating factor (GM-CSF) is a cytokine that functions as a white blood cell growth factor. GM-CSF stimulates stem cells to produce granulocytes (neutrophils, eosinophils, and basophils) and monocytes. Monocytes exit the circulation and migrate into tissue, whereupon they mature into macrophages and dendritic cells. Thus, it is part of the immune/inflammatory cascade ${ }^{(9)}$.

The pleiotropic action of cytokines includes numerous effects on cells of immune system and modulation of inflammatory response. Many characteristic features of cytokines make them particularly interesting to orthodontics. This study aimed to investigate the effects of orthodontic tooth movement on the periodontium by analyzing pro-inflammatory cytokines (IL-1 $\alpha$, IL-8 and GM-CSF) levels within saliva. 


\section{Subjects and Methods}

Eighteen subjects (12 females and 6males) were enrolled in this prospective study, their age range from (18-23years). They were referred to the postgraduate clinic of Orthodontic Department in the College of Dentistry/Baghdad University. The patients were selected if they fulfilled the following inclusion criteria: no smoking, no previous orthodontic treatment, no extensive dental restorations or adhesive fixed partial dentures, no periodontal disease, and no use of antibiotics during or before bonding the brackets to the teeth. All patients' rights were protected, and informed consent was obtained according to the Faculty of Dentistry Ethical Committee Board.

Oral hygiene status of the patients was determined on a separate occasion before initiating the experimental procedures. Individuals who were included in the study group were only those with a plaque index $\leq 2$ and gingival index $\leq$ $1^{(10)}$.

Tooth separation for proper seating of orthodontic molar bands through applying elastomeric separators around upper and lower first molar. After separation, the upper and lower bands would be cemented on permanent first molars through good isolation of the tooth and applying an even thickness of Glass ionomer band cement (Automix dual cure glass ionomer band cement /ASPIRE, Orthoclassic, USA). Subsequently, bonding of fixed orthodontic appliance was done including polishing of the enamel surface for 5 seconds each tooth, then rinsing with air and water spray, isolation and dryness to prepare teeth for etching using 35\% phosphoric acid for 20 seconds followed by washing and dryness until a lightly whitish appearance became evident making the teeth surface ready to receive the bonding material (Self cure orthodontic adhesive/ ASPIRE, Orthoclassic, USA) and stainless steel orthodontic bracket ( Roth 0.022“"slot/ Orthoclassic, USA) placed in exact position for each tooth.

When the bonding material is completely set, 0.014 " Nickel titanium archwire (NiTi/ Orthoclassic, USA) was inserted in the tube of the band and ligated to the brackets by means of elastomeric rings, ( latex free /Orthoclassic, USA). Then the patients were given out a kit containing an orthodontic tooth brush, interdental brush and a tube of dentifrice for standerization. All patients received special oral hygiene instructions, they were told to brush 3 times a day for 2 minutes, using an amount of dentifrice that covered the entire head of the toothbrush.

Two $\mathrm{ml}$ of whole unstimulated saliva was collected using plastic test tubes. Samples were taken at baseline (BT), before appliance was applied (considered as control); at two weeks (T2) and after 4weeks (T4) from placement of orthodontic appliance. Then saliva centrifuged at $1000 \mathrm{rpm}$ for 10 minutes; this was done within 1hour after collection to eliminate debris and cellular matter, the supernatants were aspirated immediately, divided into aliquots and kept at $-20^{\circ} \mathrm{C}$ until used for assay. Levels of IL-1 $\alpha$, IL-8 and GM-CSF have been estimated by using commercially available enzyme-linked immunosorbent assay (ELISA) and performed as recommended in leaflet with kits (Immunotech, France).

Statistical analysis: Comparison of salivary IL-1 $\alpha$, IL-8 and GM-CSF levels among groups was calculated by Kruskal-Wallis-test and Mann-Whitney-test. $\mathrm{P}$ values of $\mathrm{P}<0.001$ and $\mathrm{P}<0.05$ were considered significant.

\section{Result}

The current results revealed no statistically significant differences in the levels of IL-1 $\alpha$ at any times $\mathrm{P}>0.05$, as clearly shown in table (1). On the other hand, significant elevation in median salivary levels of IL-8 and GM-CSF was found after 2 weeks and after 4 weeks from placement of orthodontic appliance as compared to their levels at baseline time, $\mathrm{P}<0.01$. In addition the levels of these cytokines were significantly decrease at 4 weeks when compared to their levels at 2 weeks $\mathrm{P}<0.01$, table $(1 \& 2)$.

\section{Discussion}

Orthodontic tooth movement occurs by the remodeling of the alveolar bone as a result of the force exerted on the periodontium ${ }^{(11)}$. Endothelial cells are activated and cytokines and chemoattractants that result in leukocyte activation are produced ${ }^{(12)}$. This interaction between endothelial cells and leukocytes is important in the inflammatory process, as it produces proinflammatory cytokines and antiinflammatory cytokines (13). Proinflammatory cytokines play important roles in bone and root resorption. Determining the levels of various cytokines during various phases of orthodontic treatment undoubtedly contributes to our understanding of the underlying mechanisms of tooth movement.

The current results revealed no significant differences in median salivary level of IL-1 $\alpha$ before and after placement of orthodontic appliance, this finding is consistent with other study reported by Hyoung-Seon Baik and colleagues who reported that there was no significant difference in the expression of IL-1 $\alpha$ before and after placement of orthodontic attachments ${ }^{(14)}$. This may be due to the fact that IL-1 $\alpha$ appears to express during a very early stage of orthodontic movement, which may explain the lack of an increase IL-1 $\alpha$ at 2 weeks and 4 weeks in our study.

Another interesting finding in the present study was the significant elevation in median salivary levels of IL-8 and GM-CSF after 2 weeks and after 4 weeks from placement of orthodontic appliance as compared to their levels at baseline time. This is in accordance with other studies $(1,15,16,17)$ who showed the acute 
inflammatory response in the initial phase of tooth movement. Tuncer et al., 2005 reported increased levels of IL-8 at PDL tension sites and proposed it to be a triggering factor for bone remodeling ${ }^{(17)}$. Because periodontal tissues are remodeled at both tension and pressure sites during tooth movement, the increased amount of IL-8 at both sites just after application of mechanical forces might be a sign of neutrophil reaction in the area. After the acute response, IL- 8 stimulation might be continued at the tension sites. So they concluded that under the influence of mechanical forces, pressure sites indirectly contribute to the production of IL-8. Thus, the production of IL-8 is regulated differentially at tension and pressure sites and probably plays a major role in the initial stage of remodeling ${ }^{(15)}$. On the other hand, Pinkerton and associates pointed out to that a GMCSF gene was found to be consistently upregulated after placement of orthodontic appliance, and this lead to release of this cytokin ${ }^{(18)}$. In contrast with these results, Wilson observed that there was no statistically significant difference in the levels of GM-CSF between any times at any sites ${ }^{(19)}$. Moreover, Alhashimi et al., 2001 have shown that tumor necrosis factor alpha directly stimulates the differentiation of osteoclast progenitors to osteoclasts in the presence of GM-CSF ${ }^{(20)}$. In conclusion these findings suggest that orthodontic force induces release of the proinflammatory cytokines (IL- 8 and GM-CSF), in addition proinflammatory cytokines play a crucial role in bone resorption after the application of orthodontic force.

\section{References}

[1] Jan G, Wim T, Marc Q, Sofie St. Longitudinal changes in gingival crevicular fluid after placement of fixed orthodontic appliances. Am J Orthod Dentofacial Orthop. 2011; 139: 735-44

[2] Krishnan V, Davidovitch Z. Cellular, molecular, and tissue-level reactions to orthodontic force. Am J Orthod Dentofacial Orthop. 2006; 129(4):469.

[3] Bartzela T, Türp JC, Motschall E, Maltha JC. Medication effects on the rate of orthodontic tooth movement: a systematic literature review. Am J Orthod Dentofacial Orthop. 2009; 135(1):16-26.

[4] Taba M, Kinney J, Kim AS, Giannobile WV. Diagnostic biomarkers for oral and periodontal diseases. Dental Clinics of North America. 2005; 49(3):551-71.

[5] Stoycheva MS, Murdjeva MA. Correlation between serum levels of interleukin 1- $\beta$, interleukin 1-ra, interleukin-6, interleukin 10, interleukin 12 , tumor necrosis factor- $\alpha$ and interferon- with some clinical and laboratory parameters in patients with salmonellosis. Biotechnol Equip. 2005;19:143-6.

[6] Nicklin MJ, Weith A, Duff GW. A physical map of the region encompassing the human interleukin-1 alpha, interleukin-1 beta, and interleukin-1 receptor antagonist genes. Genomics. 1994; 19 (2): 382-4.

[7] Bankers-Fulbright JL, Kalli KR, McKean DJ. Interleukin-1 signal transduction. Life Sci. 1996; 59 (2): 61-83.

[8] Bickel M. The role of interleukin-8 in inflammation and mechanisms of regulation. J Periodontol. 1993; 64:456-60.

[9] Hamilton JA. GM-CSF in inflammation and autoimmunity. Immunology. 2002; 23(8): 403-8.

[10] Loe H, Silness J. Periodontal disease in pregnancy I. Prevalence and severity. Acta Odontol Scand. 1963; 21: 533-51.

[11] Davidovich Z. Tooth movement. Critical reviews in Oral Biology and Medicine. 1991; 21: 411-50.

[12] Cooper SM, Sims MR. Evidence of acute inflammation in the PDL subsequent toorthodontic tooth movement in rats. Australian Orthodontic Journal. 1989; 11: 107-9.

[13] Maroszynska I, Fiedor P. Leukocytes and endothelial interaction in as rate limiting step in the inflammatory response and a key factor in the ischaemia reprofusion injury. Annals of transplantation. 2000; 5: 5-11.

[14] Baik HS, Kim C, Lim WH, Chun Y. Interleukin-1 $\alpha$ and tumor necrosis factor- $\alpha$ expression on the compressed side of gingiva during orthodontic tooth movement. Open Journal of Stomatology. 2012; 2:182-7.

[15] Kaya1 FA, Hamamci N, Basaran G, Dogru M, Yildirim TT. TNF- $\alpha$, IL- $1 \beta$ and IL-8 levels in tooth early leveling movement orthodontic treatment. J Int Dent Med Res. 2010; 3 (3):116-21.

[16] Ingman T, Apajalahti S, Mantyla P, Savolainen P, Sorsa T: Matrix metalloproteinase-1 and 8 in gingival crevicular fluid during orthodontic tooth movement. Eur J Orthod. 2005;27:202-7.

[17] Tuncer BB, Ozmeriç N, Tuncer C, Teoman I, Cakilci B, Yücel A, Alpar R, Balo K. Levels of interleukin-8 during tooth movement. Angle Orthod. 2005; 75: 539-44.

[18] Pinkerton MN, Wescott DC, Gaffey BJ, Beggs KT, Milne TJ, Meikle MC. Cultured human periodontal ligament cells constitutively express multiple osteotropic cytokines and growth factors, several of which are responsive to mechanical deformation. J Periodont Res. 2008; 43: 343-51.

[19] Wilson JL. Analysis of biological pathways, associated with orthodontic force, using multiplex arrays. A thesis submitted to the University of Birmingham for the degree of master of philosophy. 2011.

[20] Alhashimi N, Frithiof L, Brudvik P, Bakhiet M. Orthodontic tooth movement and de novo synthesis of proinflammatory cytokines. Am J Orthod Dentofacial Orthop. 2001; 119 (3):307-12.

Table-1: Median levels of Salivary IL-1 $\alpha(\mathrm{pg} / \mathrm{ml})$.

\begin{tabular}{||l|c|c|c|c||}
\hline Cytokines & Baseline & at 2 wks & at 4 wks & P (Kruskall-Wallis) \\
\hline Minimum & 0 & 3 & 3 & \\
\hline Maximum & 15 & 20 & 19 & $>0.05$ \\
\hline Median & 5 & 18 & 18 & \\
\hline Number & & & & \\
\hline P Mann-Whitney) & & & & \\
\hline BT x T2= NS & & & & \\
\hline T2 X T4= NS & & & \\
\hline
\end{tabular}


Evaluation of Salivary Levels of Proinflammatory Cytokines (IL-1 $\alpha, I L-8$ and GM-CSF) in Adult

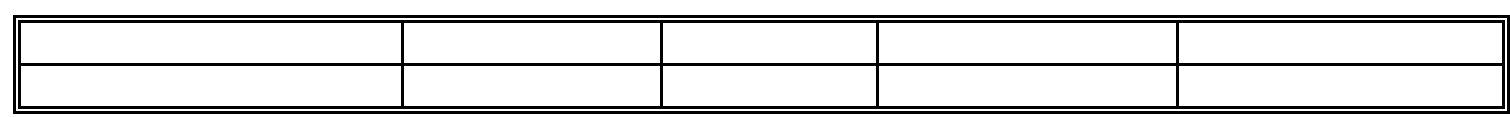

Table-2: Median levels of Salivary IL-8 (pg/ml).

\begin{tabular}{|c|c|c|c|c|}
\hline Cytokines & Baseline & at 2 wks & at 4 wks & P (Kruskall-Wallis) \\
\hline Minimum & 40 & 75 & 50 & \\
\hline Maximum & 110 & 200 & 150 & \\
\hline Median & 45 & 80 & 55 & $<0.01$ \\
\hline Number & 18 & 18 & 18 & \\
\hline \multicolumn{5}{|c|}{ P (Mann-Whitney) } \\
\hline \multicolumn{5}{|l|}{$\mathrm{BT} \times \mathrm{T} 2=<0.01$} \\
\hline \multicolumn{5}{|l|}{$\mathrm{T} 2 \mathrm{X} \mathrm{T} 4=<0.05$} \\
\hline & & & & \\
\hline & & & & \\
\hline
\end{tabular}

Table-3: Median levels of Salivary GM-CSF (pg/ml).

\begin{tabular}{||l|c|c|c|c||}
\hline \hline Cytokines & Baseline & at 2 wks & at 4 wks & P (Kruskall-Wallis) \\
\hline Minimum & 0 & 5.1 & 5 & \\
\hline Maximum & 22 & 60 & 30 & \\
\hline Median & 6.2 & 19 & 13 & $<0.01$ \\
\hline Number & 18 & 18 & 18 & \\
\hline P $($ Mann-Whitney) & & & & \\
\hline BT x T2= <0.01 & & & & \\
\hline T2 X T4= $<\mathbf{0 . 0 5}$ & & & & \\
\hline & & & & \\
\hline
\end{tabular}

